\title{
BRIEF
}

\section{A Simulation Activity to Assess Student Pharmacists' Knowledge and Perceptions of Oncology Pharmacy}

\author{
Elizabeth Ledbetter, PharmD, ${ }^{a}$ Scarlet Lau, PharmD, MPH, ${ }^{\mathrm{b}}$ Andrea Enterline, PharmD, ${ }^{\mathrm{c}}$ \\ Bethany Sibbitt, PharmD, ${ }^{\mathrm{d}}$ Aleda M. H. Chen, PharmD, PhD ${ }^{\mathrm{d}}$ \\ ${ }^{a}$ IU Health Advanced Therapies Pharmacy, Indianapolis, Indiana \\ ${ }^{\mathrm{b}}$ West Penn Hospital/Forbes Hospital, Pittsburgh, Pennsylvania \\ ${ }^{\mathrm{c}}$ CareSource, Dayton, Ohio \\ ${ }^{\mathrm{d}}$ Cedarville University School of Pharmacy, Cedarville, Ohio \\ Submitted December 14, 2018; accepted October 31, 2019; published May 2020.
}

Objective. To assess the impact of an interactive activity on student pharmacists' confidence in answering oncology-related questions and their perceptions of an oncology pharmacists' roles in practice. Methods. Two cohorts (2016 and 2017) of third-year student pharmacists completed a two-hour, interactive, four-station activity during the fourth week of an oncology module. Each station simulated a different oncology-related scenario that represented a specific practice settings. Pre- and post-activity surveys were administered to determine changes in students' confidence levels and perceptions.

Results. Over the two years, 66 student pharmacists completed the pre- and post-activity survey instruments. In both cohorts, there was a significant increase in scores on all items regarding students' confidence. Student pharmacists' perceptions of pharmacists' roles also improved significantly.

Conclusion. The simulation activity was effective as confirmed by improvement in student pharmacists' post-activity scores on confidence and perception. Determining student pharmacists' comfort in responding to oncology scenarios is important to prepare them for practice in any setting.

Keywords: oncology practice, student pharmacist, confidence, perception, chemotherapy

\section{INTRODUCTION}

Pharmacists' roles in the care of oncology patients are continually evolving as providers are challenged with medication shortages, rapid availability of newly approved oncolytics (oral chemotherapy), high-cost therapies, and reduced reimbursement. According to studies by the American Society of Clinical Oncology, the demand for oncology services may rise disproportionately to other services because of patient age distribution, provider practice patterns, and the number of oncology fellowship positions for medical graduates available in the United States. ${ }^{1,2}$ Pharmacists are well-positioned to compensate for the predicted shortage of providers in this area because of their extensive pharmacology knowledge as well as the postgraduate residency training and advanced specialty certification available in this area.

Oncology pharmacists are responsible for comprehensive medication management; counseling patients; developing therapeutic plans; evaluating drug therapies;

Corresponding Author: Bethany Sibbitt, Cedarville University School of Pharmacy, 251 N. Main St., Cedarville, OH 45314. Tel: (937) 766-7492. Fax: 937-766-7410. Email: bgsibbitt@cedarville.edu. monitoring treatment-related toxicities; ensuring safe compounding and preparation of chemotherapies, biologics, and immunotherapy; and serving as an integral member of oncology interdisciplinary teams. ${ }^{2}$ Pharmacists also influence formulary management and institutional policies and standards. ${ }^{3}$ As treatment options become more targeted toward tumor type, biomarkers, and mutation expression, pharmacists can play a vital role in therapy optimization. Pharmacy-led drug evaluation can subsequently lead to decreased costs, prevention of adverse events, and improved outcomes and quality of life. Growing recognition of pharmacists' value supports the continual need for adequate training in oncology pharmacotherapy to deliver quality patient care. ${ }^{3,4}$ To achieve this, it is critical to prepare student pharmacists as future practitioners equipped to address the provider gap in oncology care.

There are few studies in the literature on the implementation of pharmacy oncology courses or advanced pharmacy practice experiences (APPE) in oncology and their impact on student pharmacists' knowledge and perceptions of oncology practice. In a study evaluating the impact of an oncology elective course on student 


\section{American Journal of Pharmaceutical Education 2020; 84 (5) Article 7474.}

pharmacists' interest in oncology practice, only 45 of 53 students completed two consecutive semesters of the course. There was a decrease in the number of students showing interest in pursuing oncology specialty residencies or becoming board certified in oncology. ${ }^{5}$ Another study examined the impact of a one-credit hour oncology elective course on examination scores for a pharmacotherapeutics course between third-year student pharmacists who took the oncology elective course and those who did not. Students who took the elective had significantly higher scores on three out of the six examinations. Students also provided positive feedback and expressed an increased interest in pursuing a career in oncology pharmacy, with $35 \%$ agreeing that the course increased their interest in oncology. ${ }^{6}$ In a third study evaluating the impact of a comprehensive ovarian cancer case-based simulation on knowledge in 109 third-year student pharmacists, a significant improvement in knowledge was seen as evidenced by higher scores on three of the six items on the post-activity test compared to the pre-activity test. The students' perceptions of oncology pharmacists' roles and students' self-confidence in reviewing and dispensing oncolytics also increased significantly. ${ }^{7}$ Another study reported on an acute care oncology pharmacy practice experience in a layered learning practice model for APPE students, PGY1 residents, and PGY2 residents to evaluate learner perception of the model and achievement of knowledge-based learning objectives. All posttest scores significantly increased compared to pretest scores, and learners viewed the experience as positive and felt it allowed for improvement in clinical and self-management skills. ${ }^{8}$ Most of the studies discussed here documented improvement in pharmacy students' oncology knowledge after implementation of an oncology APPE or elective course and positive perceptions of oncology pharmacy practice; however, because these were elective experiences, they only impacted those student pharmacists who enrolled in the course.

There are a limited number of studies in the literature that discuss student pharmacists' perceptions about their use of oncology knowledge once they are licensed pharmacists. In a study published in 2017, researchers evaluated Florida student pharmacists' interests $(\mathrm{N}=532$ from five out of six schools of pharmacy) in oncology pharmacy and areas of oncology specialization. Three-fourths $(75 \%)$ of students were only moderately comfortable or not comfortable with oncology, while over half $(56 \%)$ of students expressed interest in pursuing oncology in their future pharmacy practice. Most students reported no access to oncology-related experiences, with less than five students reporting having earned experiential hours related to oncology. They also requested additional oncology experiential opportunities and elective courses. Therefore, although some students showed interest in learning more about or pursuing a career in oncology, most students identified a perceived need for further training in oncology topics. ${ }^{9}$

Although studies have been published that discuss improvements in student pharmacists' oncology knowledge based on pre- and post-activity test scores and perceptions of oncology practice at the end of interventions, there are few studies that have evaluated student confidence in their knowledge of oncology pharmacotherapy and compared their perceptions of oncology practice before and after participating in interactive activities in a required oncology module. We address these gaps in the study presented here. The objectives of this study were to assess students' confidence in answering oncologyrelated questions in different pharmacy practice settings pre- and post-activity and students' perceptions of the importance of pharmacists in the treatment of cancer patients prior to the course and after participating in the interactive activity.

\section{METHODS}

This study was approved by the Institutional Review Board (IRB) of Cedarville University. Two cohorts of students (fall 2016 and fall 2017) were included.

Oncology was taught during a five-week module in the fall of the third professional year. In the first professional (P1) year, students were introduced to pharmacist roles and responsibilities through an integrated approach between the didactic (Introduction to Pharmacy Practice and Pharmacy Practice Laboratory courses) and the experiential curriculum, which consisted of introductory pharmacy practice experiences (IPPEs) in community pharmacies. This was reinforced in the second professional year with IPPEs in the institutional setting. In the oncology module, basic, clinical, and social and administrative sciences were integrated during the module, and key oncologic, hematologic, and palliative care concepts were included primarily through team-based learning (TBL) pedagogy. Cases were also incorporated throughout the module to reinforce concepts as part of TBL or independently. Prior to beginning the module, students had limited exposure to oncologic pharmacotherapy, pathophysiology, and therapeutics, as well as limited training in the pharmacist's role in patient care, dispensing, and medication safety. Based on feedback from students, there was a gap in understanding of the importance of having knowledge of oncology across practice settings. To address this issue and reinforce the roles and responsibilities of pharmacists introduced in the $\mathrm{P} 1$ year, a prior 


\section{American Journal of Pharmaceutical Education 2020; 84 (5) Article 7474.}

chemotherapy checking activity was chosen to enhance and provide students with a better idea of how oncology impacts multiple practice settings.

A four-station activity was created to represent different practice settings: managed care, community pharmacy, a hospital that does not have a clinical pharmacist in oncology, and a hospital that does have a clinical pharmacist in oncology. To create these stations, pharmacists in each of these settings were interviewed regarding their roles and responsibilities with oncology-related medications. The information from those interviews was used to create scenarios intended to highlight the responsibilities of the pharmacists in each setting. Students then practiced those responsibilities. In scenario 1 (managed care), a cost-minimization research abstract was provided to students. Students used the information in the article in addition to electronic drug information resources to examine the cost and efficacy of an oncology medication for formulary management. In scenario 2 (community pharmacy), a patient presented to the pharmacy with adverse effects from chemotherapy (sleep disturbances, constipation), and the students had to make non-pharmacologic recommendations that did not interact with the patient's chemotherapy regimen. In scenario 3 (hospital without an oncology pharmacist), the students had to determine the best course of action for a patient presenting for cancer-related surgery who was enrolled in an oncology clinical drug trial (when to discontinue the medication, when to restart) and develop a communication plan for the surgical team. In scenario 4 (hospital with oncology pharmacist), students had to verify four prescriptions, which required them to use their compounding and calculations skills. Students completed the stations in pre-assigned teams, and had 75 minutes to complete all four stations. The last 30 minutes of the class was allotted for a facilitator-led discussion of the students' answers. The activity took place in week four of the module during a two-hour class session.

At this point in the curriculum, students had learned the pharmacology of oncology medications as well as about most major solid tumors. Thus, this activity was intended to build on the prior knowledge they had gained and solidify their understanding of the role of pharmacists in various oncology settings. Students worked in preassigned teams (five to six students per team), completed each of the four stations, and then participated in a debrief. The same faculty member (a social and administrative sciences faculty member who also was a pharmacist with experience as a community pharmacist and as a clinical pharmacist in managed care) facilitated the activity for both student cohorts, with a fourth professional year student who was completing an advanced pharmacy practice experience (APPE) co-facilitating. Students could spend as much time as needed at each station within the designated 75 minutes. The facilitators were available for questions and led the debrief based on a key at the end of the session.

To address the objectives, the same survey instrument (17 items rated on a seven-point Likert-type) was administered at the beginning of the oncology module (pre-activity survey) and again immediately after students completed the activity (post-activity survey). While completion of the module itself would likely impact the outcomes, the research team wanted to identify changes in student confidence and perceptions from the beginning of the course to the end of the activity as the activity built upon the knowledge and skills the students had developed over the prior weeks. The survey had two separate aspects: the first nine items assessed students' confidence in answering oncology-related questions while the remaining eight items assessed students' level of agreement with pharmacists' involvement in oncology patient care. These items were created by reviewing the literature and conducting interviews with the pharmacists as described above. Then, before implementation, the survey items underwent review by the module coordinator (a clinical oncology pharmacist) and a faculty member with survey experience.

Statistical analysis was conducted using SPSS, v. 25.0 (IBM, Armonk, NY). A $p$-value of $<.05$ was considered significant. As the data were not normally distributed, medians were calculated for the pre- and post-activity survey items. Changes in scores from pre- to post-activity survey were assessed using a Wilcoxon signed rank test. Differences between cohorts were assessed using a MannWhitney U test. Descriptive statistics were performed for all demographic items, including interquartile range (IQR, spread of the $25^{\text {th }}$ to $75^{\text {th }}$ percentiles) for medians, and differences between cohorts were assessed using a chi-square or unpaired $t$ test.

\section{RESULTS}

Sixty-six students $(100 \%$ response rate) from two cohorts completed pre- and post-activity surveys. There were no significant differences in gender $(p=.805)$, ethnicities $(p=.163)$, and past oncology experience $(p=.163)$ between cohorts. However, student pharmacists in fall 2016 were significantly younger (23.3 years vs. 24.6 years, $p=.037$; Table 1).

Students in both cohorts demonstrated significantly more confidence in answering all nine oncology-related questions in different pharmacy practice settings from pre- and post-activity (Table 2). In both cohorts, 


\section{American Journal of Pharmaceutical Education 2020; 84 (5) Article 7474.}

Table 1. Demographics of Student Pharmacists Who Participated in a Simulation Activity Intended to Improve Their Knowledge and Perceptions of Oncology Pharmacy, $\mathrm{N}=33$

\begin{tabular}{lccc}
\hline & Cohort 2016 & Cohort 2017 & $p$ Value \\
\cline { 2 - 4 } No (\%)/Mean (SD) & No (\%)/Mean (SD) & .81 \\
\hline Gender, No. (\%) & $17(51.5)$ & $18(54.5)$ & \\
$\quad$ Male & $16(48.5)$ & $15(45.5)$ & .16 \\
Female & & & \\
Ethnicity & $27(81.8)$ & $18(54.5)$ & $7(21.2)$ \\
$\quad$ Caucasian & $3(9.1)$ & $1(3.0)$ & $.04^{\mathrm{a}}$ \\
African American/African & $0(0)$ & $4(12.1)$ & .16 \\
Hispanic & $3(9.1)$ & $2(6.1)$ & \\
Asian/Pacific Islander & $0(0)$ & $24.6(3.1)$ & \\
Other & $23.3(1.2)$ & $4(12.1)$ & $29(87.9)$ \\
Age & $1(3.0)$ & $32(97.0)$ & \\
Past oncology experience & & \\
Yes & & & \\
No &
\end{tabular}

${ }^{a}$ Unpaired $t$-test was used to determine significance, defined as $p<.05$, between the two cohorts of student pharmacists

significant increases were seen between student pharmacists' pre- and post-activity median confidence scores on all nine questions $(p<.001)$. Students in the fall 2016 cohort had greater increases in confidence on four of the nine survey questions than did students in the fall 2017 cohort.

Significant increases in students' perceptions of the importance of pharmacists in the treatment of cancer patients pre- and post-activity occurred in both cohorts (Table 2). In fall 2016, student pharmacists only exhibited significantly higher levels of agreement $(p<.05)$ on four of the eight questions. Students in fall 2017 had significant increases in scores on three of the same items as the 2016 cohort, but did not have significantly different scores on the fourth item (managed care pharmacists being equipped to make chemotherapy formulary recommendations). Similar increases in knowledge between the pre- and postactivity survey occurred in both cohorts.

\section{DISCUSSION}

Oncology elective courses within the pharmacy curriculum have been described in literature as increasing student pharmacists' interests in oncology pharmacy. These courses also improve students' test scores on their knowledge of oncology and understanding of oncology practice. However, there are few studies evaluating student pharmacists' confidence and perceptions of oncology pharmacy while also assessing knowledge of oncology and oncology pharmacy practice. ${ }^{6,7}$ Our study was conducted to explore student pharmacists' confidence in and perceptions of oncology pharmacy before and after completing a four-part interactive activity before the oncology course and a two-hour session in the fourth week of the course. These interventions resulted in significant improvements in scores on several items.

The interactive activity described in this study allowed students to score levels of confidence in completing oncology-related tasks and levels of agreement with oncology practice both pre-course and post-activity. It served to reinforce concepts in the module and use practical examples. The activity also provided an opportunity for students to practice calculations for a compounded chemotherapy product, give recommendations regarding nonprescription medication use and supportive care for patients undergoing chemotherapy treatments, recognize chemotherapy-related drug-drug interactions and adverse effects, navigate resources to answer questions posed by members of the healthcare team or patients and family members, and make evidence-based formulary recommendations using knowledge gained in the oncology module. Implementation of the interactive activity allowed students to apply knowledge, use critical thinking skills, and answer questions posed in oncology-related scenarios. Students worked in preassigned groups, which reinforced their teamwork and communication skills. ${ }^{7}$ Students experienced a more realistic environment simulated to represent different practice settings using a four-station activity. ${ }^{7}$ The range of oncology questions asked of the students reflected real scenarios that oncology pharmacists are expected to manage and resolve as the scope of their responsibilities expands. ${ }^{7,8}$ This activity gave students a true assessment of oncology pharmacy practice in a real-world application.

Confidence in performing oncology-related activities increased among students in both cohorts as 


\section{American Journal of Pharmaceutical Education 2020; 84 (5) Article 7474.}

Table 2. Student Pharmacists' Scores on a Survey Administered Before and After Participating in a Simulation Activity Intended to Improve Their Knowledge and Perceptions of Oncology Pharmacy

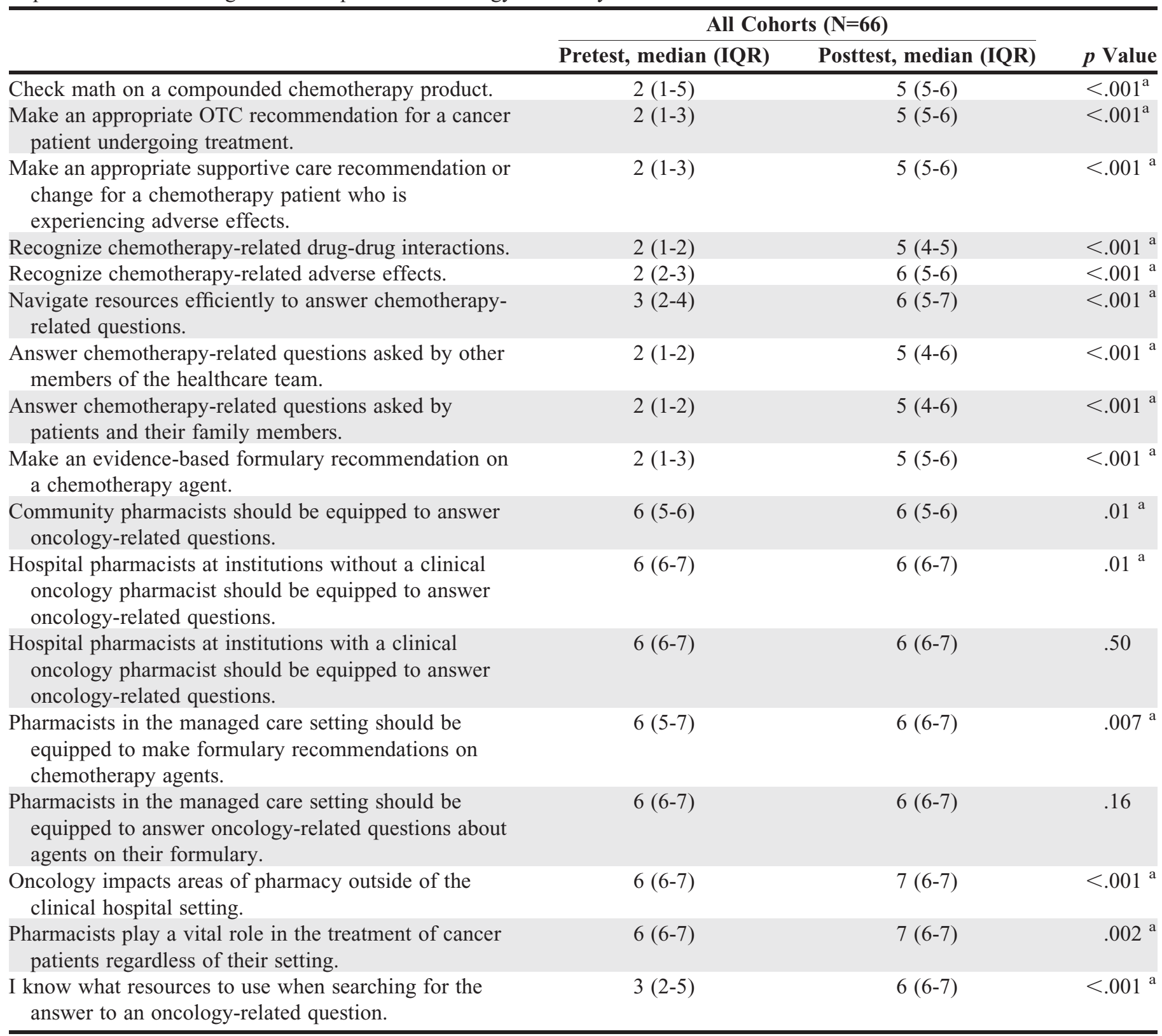

7-point Likert type scale (1=very unconfident, $7=$ very confident)

${ }^{a}$ Mann-Whitney-U test was used to compare differences in survey between pre- and post-activity as well as between cohorts

evidenced by their responses to all nine questions on the survey. Student pharmacists' perceptions of oncology pharmacists' roles also improved post-activity for all questions except one regarding hospital pharmacists being equipped to answer oncology-related questions at institutions with a clinical oncology pharmacist. Studies show that increased perceptions of confidence can lead to improvements in problem-solving skills and abilities to make clinical recommendations, which is important as pharmacists need to be competent in all areas, including in oncology. ${ }^{7}$ Knowledge gaps were identified by first administering the survey before the interactive activity that was intended to reinforce the role and importance of the oncology pharmacist in chemotherapy management.

Both cohorts of student pharmacists (2016 and 2017) had similar increases in confidence scores between the pre- and post-activity survey, which confirms that the oncology module and interactive activity were delivered consistently. Students' perception of the importance of oncology pharmacists' roles in practice also improved as evidenced by responses to all eight questions in fall 2016. 


\section{American Journal of Pharmaceutical Education 2020; 84 (5) Article 7474.}

This is supported by the literature, as past studies also saw increases in confidence scores after students completed oncology elective courses. ${ }^{6-8}$ However, in fall 2017, the increase in perceptions and improvement in scores was less than the prior cohort. Similar results were not seen in past studies which only reported increases in confidence and perception scores for all questions. ${ }^{6-8}$ The oncology module and interactive activity were administered identically in both years; therefore, the reason for the decrease in perception scores in fall 2017 is unclear and warrants further evaluation in future research.

Both cohorts took the oncology course later in the third year of the professional program, which may account for them having identical median confidence scores both pre- and post-activity. Conducting the interactive activity at the end of the third year rather than at the beginning of the PharmD program may have been the reason for the significant increases seen in confidence and agreement scores from pre- to post-activity. If administered earlier in the program, the students may not have had as much accumulated knowledge and may have had knowledge deficits prior to the course and lower increases in scores after the course. However, in a study conducted by Serag-Bolos and colleagues, both perception questions only increased by 0.7 and 1.0 points compared to 3 points in both cohorts examined. ${ }^{7}$ This may have been because the content of oncology courses varies at different institutions. Nonetheless, the elective oncology module and activity at our institution were ultimately successful in increasing student confidence and expanding perception of pharmacists' involvement in oncology. Pharmacists being able to perform oncology-related tasks is vital because of the surge in demand for oncology services. As experts in pharmacotherapy, pharmacists must know how to respond to oncology-related questions in order to provide the best patient care possible. ${ }^{2-4}$ While not predictive, students deemed to be more confident and perceptive in performing these tasks are likely to be more knowledgeable and able to critically think and assess each situation efficiently and appropriately, which are both qualities needed for students to become successful pharmacists. ${ }^{7}$ All schools of pharmacy should strive to create oncology modules with an end-goal of increasing student knowledge accompanied by appropriate confidence and perceptions.

This study had several limitations. The methods and results may not be generalizable to all student pharmacists as it involved third-year student pharmacists. Also, there was a significant difference in age between the cohorts. Students in the 2016 cohort were significantly younger than students in the 2017 cohort, which may have contributed to the greater increases found in confidence scores in this cohort. Students may have been skewed more positively due to the belief that survey responses can influence grades or perceiving that answers given may need to be acceptable, which may have led to response bias. However, pre- and post-activity assessments were created carefully by the module coordinator and a faculty member with survey experiences and were given at the beginning of the course and after the interactive activity to limit confounders. The time between the pre- and postactivity assessments does not truly inform us about which components impacted students and whether the impact was heightened by the activity as this could be due to the course itself rather than the activity presented. Lastly, this study only addressed perceptions and not knowledge or outcomes. Future work should also include administering knowledge assessments prior to the activity and administering longitudinal assessments to determine the longterm impact, particularly in APPEs and in practice.

\section{CONCLUSION}

Prior to completing an oncology module, two cohorts of student pharmacists lacked confidence in several areas of pharmacy practice. Through the implementation of an interactive oncology activity, student pharmacists' confidence in their knowledge of oncology pharmacotherapy and their perception of oncology pharmacists' roles in various practice settings increased. Student pharmacists need to be knowledgeable regarding chemotherapy regimens as all practice settings expect pharmacists to be equipped with the tools to respond to any oncology-related scenarios, regardless of whether an oncology pharmacist is present. By providing oncology pharmacotherapy education and developing awareness on the importance of knowledge and training in oncology practice through this study, students will be prepared to practice in any setting to deliver quality patient care as future pharmacists. This is important, as there is a need for pharmacists to be oncology-trained to compensate for the shortage of oncology providers. Implementation of interactive activities can assist faculty in assessing their oncology module curriculum and whether there needs to be adjustments in course content in order to allow students to be successful in oncology-related pharmacy practice. Interactive oncology activities should be implemented across all pharmacy schools to increase students' confidence and knowledge in navigating oncology-related scenarios on advanced pharmacy practice experiences or in future career practice settings.

\section{REFERENCES}

1. Erikson C, Salsberg E, Forte G, Bruinooge S, Goldstein M. Future supply and demand for oncologists: challenges to assuring access to 


\section{American Journal of Pharmaceutical Education 2020; 84 (5) Article 7474.}

oncology services. J Oncol Pract. 2007;3(2):79-86. doi: 10.1200/ JOP.0723601.

2. Holle LM, Boehnke Michaud L. Oncology pharmacists in health care delivery: vital members of the cancer care team. J Oncol Pract. 2014;10(3):e142-e145. doi: 10.1200/JOP.2013.001257.

3. Holle LM, Harris CS, Chan A, et al. Pharmacists' roles in oncology pharmacy services: results of a global survey. J Oncol Pharm Pract. 2017;23(3):185-194. doi: 10.1177/1078155216629827. 4. Leveque D, Delpeuch A, Gourieux B. New anticancer agents: role of clinical pharmacy services. Anticancer Res. 2014;34(4): 1573-1578.

5. Steinberg M, Cooper M. Impact of an oncology pharmacy elective course on guiding doctor of pharmacy students toward specializing in oncology pharmacy. Curr Pharm Teach Learn. 2015;7(5):621-626. doi: 10.1016/j.cptl.2015.06.001.

6. Nystrom KK, Pick AM. An oncology pharmacy practice elective course for third-year pharmacy students. Am J Pharm Educ. 2013; 77(1):12. doi: 10.5688/ajpe77112.

7. Serag-Bolos ES, Chudow M, Perkins J, Patel RV. Enhancing student knowledge through a comprehensive oncology simulation. Am J Pharm Educ. 2018;82(3):6245. doi: 10.5688/ajpe77112.

8. Bates JS, Buie LW, Lyons K, et al. A study of layered learning in oncology. Am J Pharm Educ. 2016;80(4):68. doi: 10.5688/ ajpe 80468 .

9. May P, Ladd J. Florida pharmacy students' perspectives on careers in oncology. J Hematol Oncol Pharm. 2017;7(2):69-75. 